Supporting information for

\title{
Electrochemical Collision of Single Silver Nanoparticles in Carbon Nanopipettes
}

\author{
Rujia Liu, Xiaoyue Shen, Dengchao Wang* \\ School of Chemical Sciences, University of Chinese Academy of Sciences, Beijing 10049, P. R. China \\ *Email: wangdengchao@ucas.ac.cn
}

\section{Table of contents:}

1. Estimation of the surface charge density of the carbon nanopipettes by numerical simulation

2. Evaluation of the collision frequency and charges of single AgNPs

Figure S1 Electrochemical characterization of prepared carbon nanopipettes

Figure S2 Size and zeta potential measurements of Ag NPs by dynamic light scattering

Figure S3 The experimental (black line) and simulated (red dots) ionic current through prepared carbon nanopipettes.

Figure S4 Current-time recordings at oxidation and reduction potential of $\pm 0.5 \mathrm{~V}$ after AgNP oxidation experiments

Figure S5. Current-time responses from single AgNPs collision at oxidation and reduction potential of \pm 0.5 V without PB buffer

Figure S6 Successive cyclic voltammograms of AgNPs inside carbon nanopipettes

Figure S7 Current-time recordings and cyclic voltammograms of $\mathrm{Ag}^{+}$reduction in carbon nanopipettes.

\section{References}




\section{Estimation of the surface charge density of the carbon nanopipettes by numerical simulation}

The surface charge density of the prepared CNPs can be obtained by fitting the experimental i-V curves. Following previous reports ${ }^{1-2}$, finite-element analysis was carried out using COMSOL Multiphysics 5.4. A 2D-axisymmetric model with "Transport of Diluted Species" and "Electrostatics" modules were used to model the mass transport processes inside CNPs. The ionic transport flux includes diffusion and migration is described by the Nernst-Planck equation, and the electric potential distribution inside the CNPs was calculated by Poisson equation.

$$
\begin{gathered}
J_{i}=-D_{i} \nabla c_{i}-\frac{z_{i} F}{R T} D_{i} \nabla c_{i} \nabla \phi \\
\nabla^{2}\left(\varepsilon_{0} \varepsilon_{\mathrm{r}} \phi\right)=-F \sum_{i} z_{i} c_{i}
\end{gathered}
$$

where $D_{i}, c_{i}$, and $z_{i}$ are the diffusion coefficient, concentration, and charge of the ionic species $i, R$ is the gas constant, $T$ is the temperature, $F$ is the Faraday constant, $\varphi$ is the potential, $\varepsilon_{0}$ and $\varepsilon_{r}$ are the vacuum permittivity and medium dielectric constant, respectively. The ionic transport current, $i_{T}$, was obtained by integrating the total flux of all the species at either reference or working electrodes. 


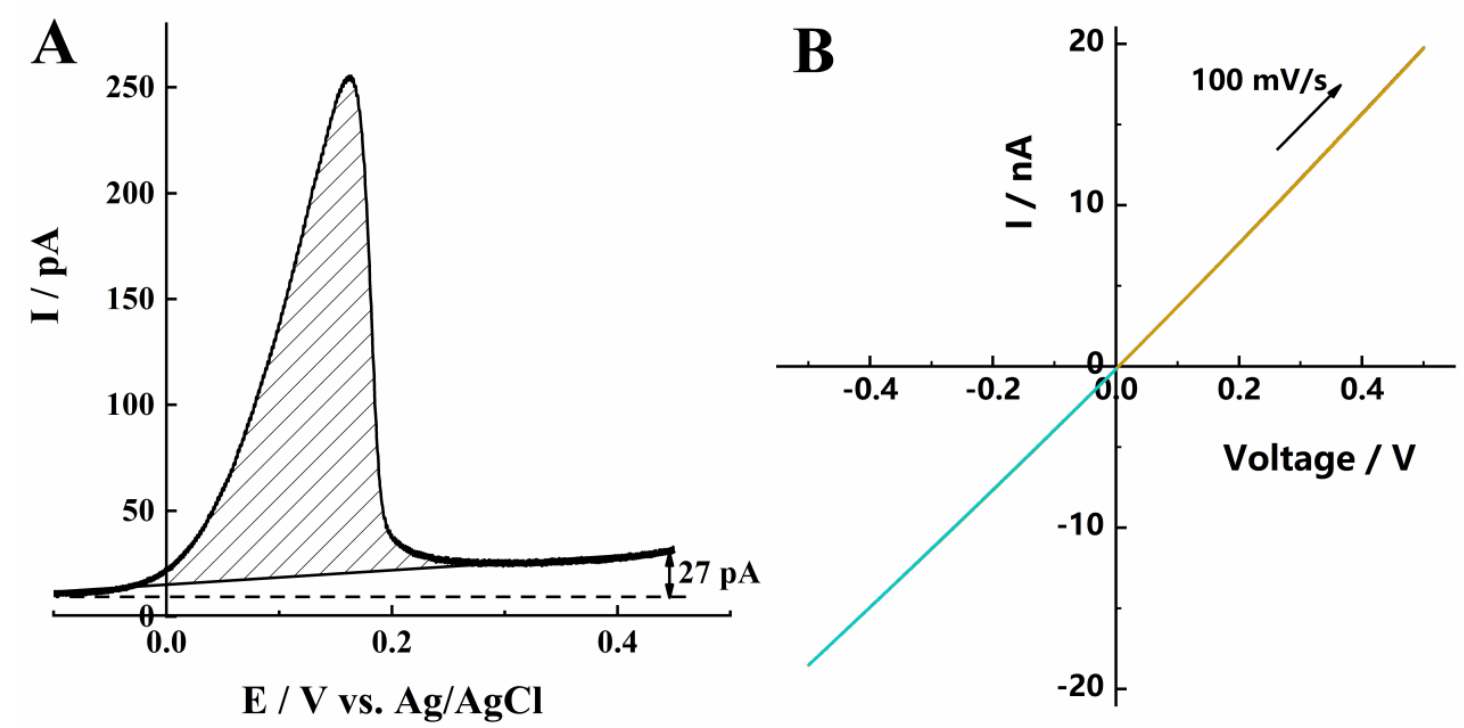

Figure S1 Electrochemical tests of the prepared carbon nanopipettes: (A) cyclic voltammetry in $1 \mathrm{mM}$ FcMeOH containing $10 \mathrm{mM} \mathrm{PB}(\mathrm{pH} 7.4)$ and (B) conductivity test in $0.1 \mathrm{M} \mathrm{KCl}$. Potential scan rate is 0.1 $\mathrm{V} / \mathrm{s}$.

The size of CNP and solution volume can be estimated from the steady-state current $\left(i_{s s}\right)$ and integrated charges $(Q)$ enclosed in the oxidation/reduction peaks. For a ring nanoelectrode, the steady state current can be expressed as $^{3}$ :

$$
i_{s s}=x 4 n F D c a
$$

Where $x$ is about $1, n=1$ is the number of transferred electrons for the FcMeOH oxidation, $F=96485$ $\mathrm{C} / \mathrm{mol}$ is the Faraday constant, $c=1 \mathrm{mM}$ and $D=7.6 \times 10^{-7} \mathrm{~cm}^{2} / \mathrm{s}$ is the concentration and diffusion coefficient of FcMeOH. From Figure S1, the diffusion-limiting current is $27 \mathrm{pA}$, and thus the radius of the CNP orifice is estimated to be $92 \mathrm{~nm}$, which is in good agreement with the TEM images (Figure 1A)

With conical cavity geometry, the volume and depth $(d)$ of solution inside CNP can be calculated from the total transferred charges during the oxidation/ reduction processes of FcMeOH by equation S4:

$$
V=\frac{Q}{F * C}=\frac{S}{v F C}=\frac{1}{3} \pi d\left(r_{1}^{2}+r_{2}^{2}+r_{1} r_{2}\right)
$$

where $S / v=285 \mathrm{pC}$ is the transferred charge $(Q)$ of $\mathrm{FcMeOH}$ oxidation, $S$ is the area of oxidation peak (shaded area in Fig S1A), and $v=0.1 \mathrm{~V} / \mathrm{s}$ is the scanning rate, so that the solution volume $V=2.95 \mathrm{pL}$ is obtained. With $r_{1}=100 \mathrm{~nm}$, half-cone angle $\theta$ of 5-1 $1^{\circ}$, and $r_{2}=r_{1}+d \tan \theta$, the estimated solution depth is $64-41 \mu \mathrm{m}$.

The transport ionic current were also recorded at various voltages to estimate the size of the fabricated 
pipettes. In the conductivity measurements, the CNP was filled with and immersed in the same solution $(0.1 \mathrm{M} \mathrm{KCl})$ and two $\mathrm{Ag} / \mathrm{AgCl}$ electrodes were put inside and outside of the CNPs to apply the voltage and collect the ionic current. At high ionic strength of $0.1 \mathrm{M} \mathrm{KCl}$, the surface charges have negligible effect on the ionic current, and thus the current is mainly determined by the volumetric solution conductivity. Shown in Figure S1B, the current-voltage curve is linear without obvious rectification. Then the size of $\mathrm{CNP}$ can be calculated by equation $\mathrm{S}^{4}$ :

$$
R=\frac{1}{\kappa \pi \operatorname{atan} \theta}+\frac{1}{4 \kappa a}
$$

Where the $\mathcal{\kappa}=0.01288 \mathrm{~S} / \mathrm{cm}$ is solution conductivity of $0.1 \mathrm{M} \mathrm{KCl}, R$ is the total resistance, $\theta=11^{\circ}$ is the angle of CNP, and $a$ is the radius CNP orifice which is $\sim 100 \mathrm{~nm}$. 
A Results

$\begin{array}{rlllll} & & & \text { Size (d.n... } & \% \text { Intensity: } & \text { St Dev (d.n... } \\ \text { Z-Average (d.nm): } & 32.83 & \text { Peak 1: } & 48.29 & 100.0 & 25.83 \\ \text { Pdl: } & 0.284 & \text { Peak 2: } & 0.000 & 0.0 & 0.000 \\ \text { Intercept: } & 0.860 & \text { Peak 3: } & 0.000 & 0.0 & 0.000 \\ \text { Result quality } & \text { Refer to quality report } & & & \end{array}$

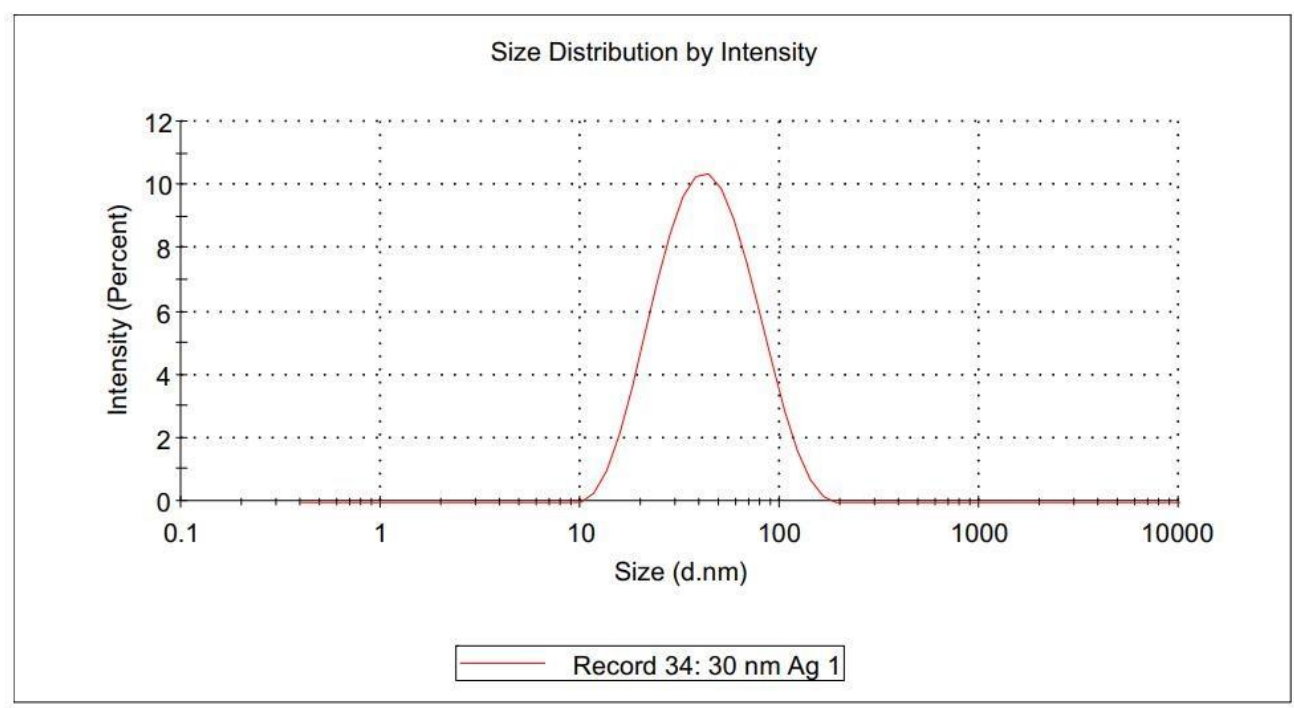

B Results

\begin{tabular}{|c|c|c|c|c|c|}
\hline & & & Mean $(m V)$ & Area $(\%)$ & St $\operatorname{Dev}(\mathrm{mV})$ \\
\hline Zeta Potential (mV): & -37.0 & Peak 1: & -38.4 & 94.3 & 10.2 \\
\hline Zeta Deviation (mV): & 12.2 & Peak 2: & -6.28 & 5.5 & 3.97 \\
\hline Conductivity $(\mathrm{mS} / \mathrm{cm})$ : & 0.841 & Peak 3: & -75.7 & 0.2 & 1.64 \\
\hline
\end{tabular}

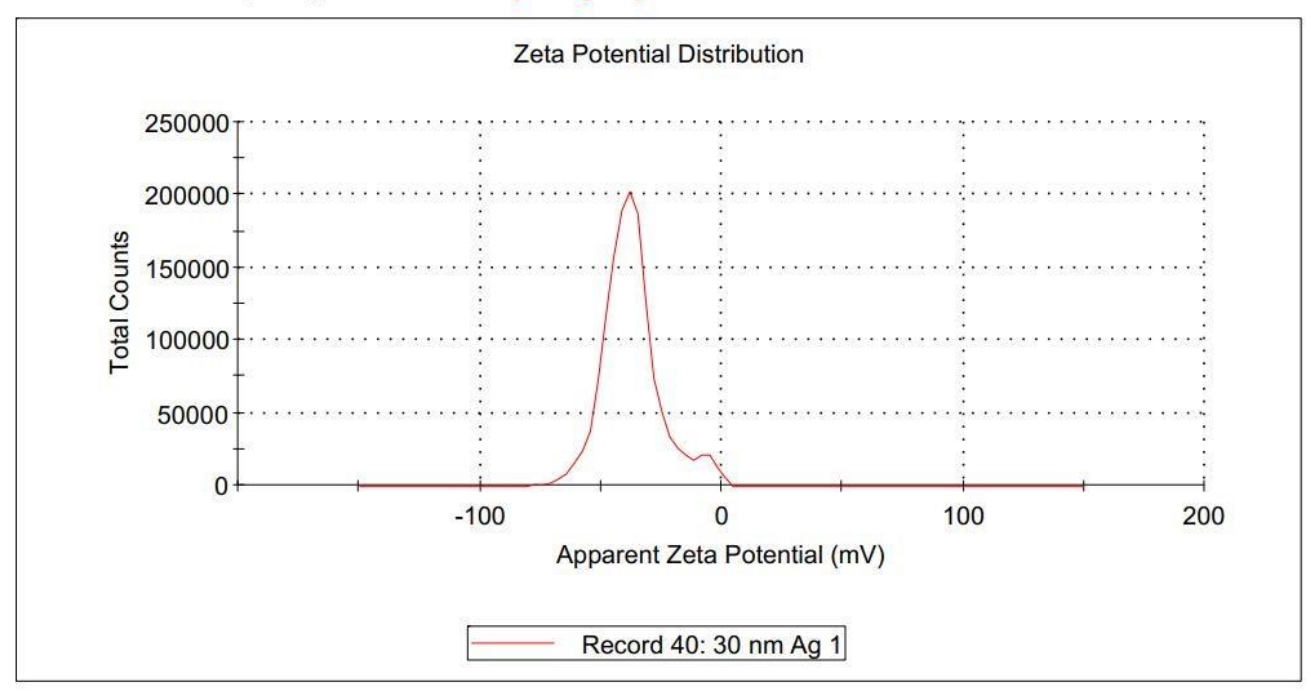

Figure S2. The size (A) and zeta potential (B) of the AgNPs characterized by dynamic light scattering. 


\section{Evaluation of the collision frequency and charges of single AgNPs}

2.1 Collision Frequency calculation: The concentration of Ag NPs was 25 pM containing $10 \mathrm{mM}$ $\mathrm{NaNO}_{3}$ and $10 \mathrm{mM}$ PB. In total, there were 485 events in $198 \mathrm{~s}$, and the collision frequency is about 2.5 $\mathrm{Hz}$. The theoretical frequency based on the diffusion model is $0.1 \mathrm{~Hz}$ calculated by equation $\mathrm{S} 6$ and $\mathrm{S}^{5}$

$$
f=4 D_{N P} C_{N P} a N_{A}
$$

Where $C_{N P}=25 \mathrm{pM}$ is the number concentration of Ag NPs, $N_{A}=6.02 \times 10^{23} / \mathrm{mol}$ is the Avogadro's number. $D_{N P}=1.629 \times 10^{-11} \mathrm{~m}^{2} / \mathrm{s}$ is the diffusion coefficient of Ag NPs, which can be calculated by eq S5.

$$
D_{N P}=\frac{k_{B} T}{6 \pi \eta a_{N P}}
$$

Where $k_{B}=1.381 \times 10^{-23} \mathrm{~J} / \mathrm{K}$ is the Boltzmann constant, $T=298 \mathrm{~K}$ is the temperature, $\eta=8.94 \times 10^{-4}$ Pa.s at $298 \mathrm{~K}$ is the dynamic viscosity.

\subsection{Charge estimation of single AgNPs:}

For a fully oxidized AgNP, the total transferred charges can be estimated as:

$$
Q=\frac{Z F}{A_{r}} \cdot \rho \cdot \frac{4}{3} \pi r^{3}
$$

where $z=1$ is transfer electron number, $A_{r}=107.9$ is the atomic mass of silver, $\rho=10.49 \mathrm{~g} / \mathrm{cm}^{3}$ is the density of silver, and $Q=0.13 \mathrm{pC}$ is the total charge transferred during an $\mathrm{Ag} \mathrm{NP}$ oxidation.

Interestingly, instead of diffusing away from the $\mathrm{CNP}$, the produced $\mathrm{Ag}^{+}$is found to accumulate in the inner cavity of CNP. The amount of produced $\mathrm{Ag}^{+}\left(n_{\mathrm{Ag}}\right)$ from an $\mathrm{Ag} \mathrm{NP}$ oxidation is $1.34 \times 10^{-18} \mathrm{~mol}$, so the equivalent concentration (c) of $\mathrm{Ag}^{+}$in $\mathrm{CNP}$ is $0.45 \mu \mathrm{mol} / \mathrm{L}$, with a solution volume of $V=2.95 \mathrm{pL}$. (Equation S9)

$$
c=\frac{n_{A g^{+}}}{V}=\frac{Q / F}{V}
$$




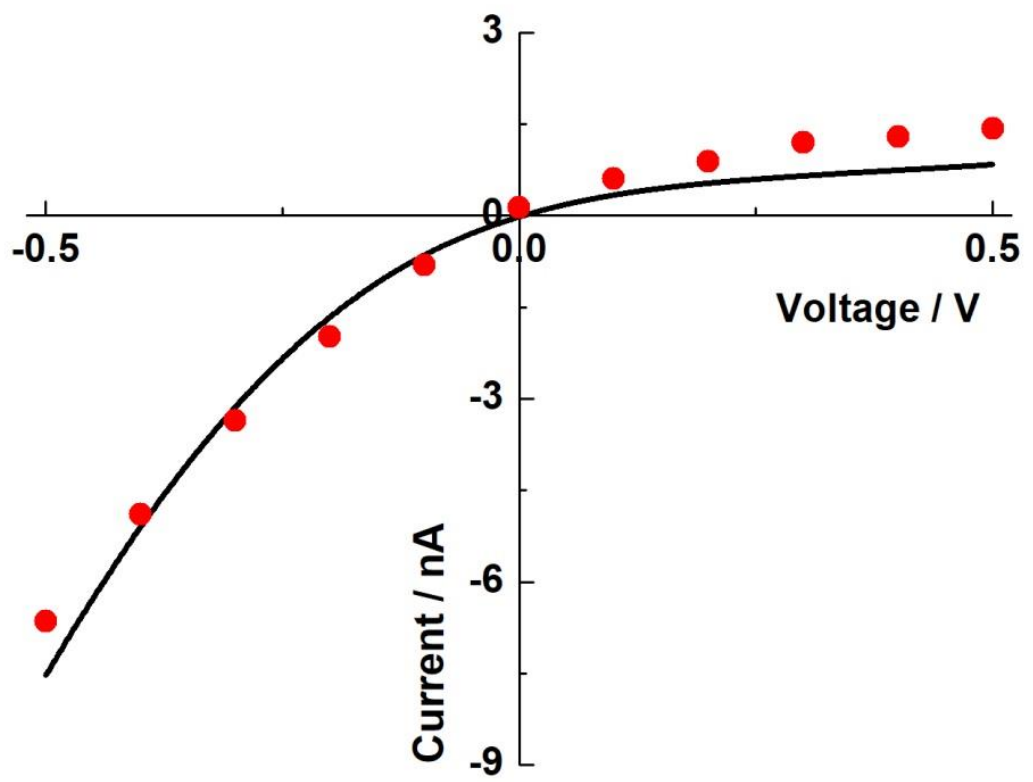

Figure S3. The experimental (black line) and simulated (red dots, fitted $\sigma=-0.10 \mathrm{C} / \mathrm{m}^{2}$ ) ionic current through prepared carbon nanopipettes. Solution contains $10 \mathrm{mM} \mathrm{NaNO}_{3}+10 \mathrm{mM} \mathrm{PB}$ buffer $(\mathrm{pH} 7.4)$. The positive voltages are defined by inside versus outside. 


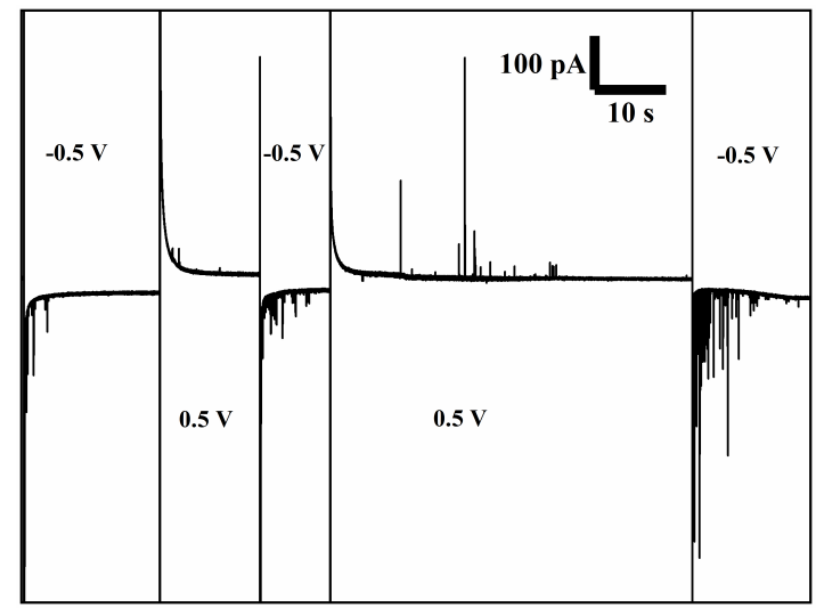

Figure S4. Current-time responses at oxidation and reduction potential of $\pm 0.5 \mathrm{~V}$ after AgNP oxidation experiments. Conditions: $25 \mathrm{pM} \mathrm{Ag} \mathrm{NPs,} 10 \mathrm{mM} \mathrm{NaNO}_{3}, 10 \mathrm{mM}$ PB (pH 7.4), the sampling rate is 10 $\mathrm{kHz}$ and the low-pass filter frequency was $3 \mathrm{kHz}, r_{\mathrm{NP}}=15 \mathrm{~nm}, a=100 \mathrm{~nm}, E=0.5 \mathrm{Vvs} . \mathrm{Ag} / \mathrm{AgCl}$ 


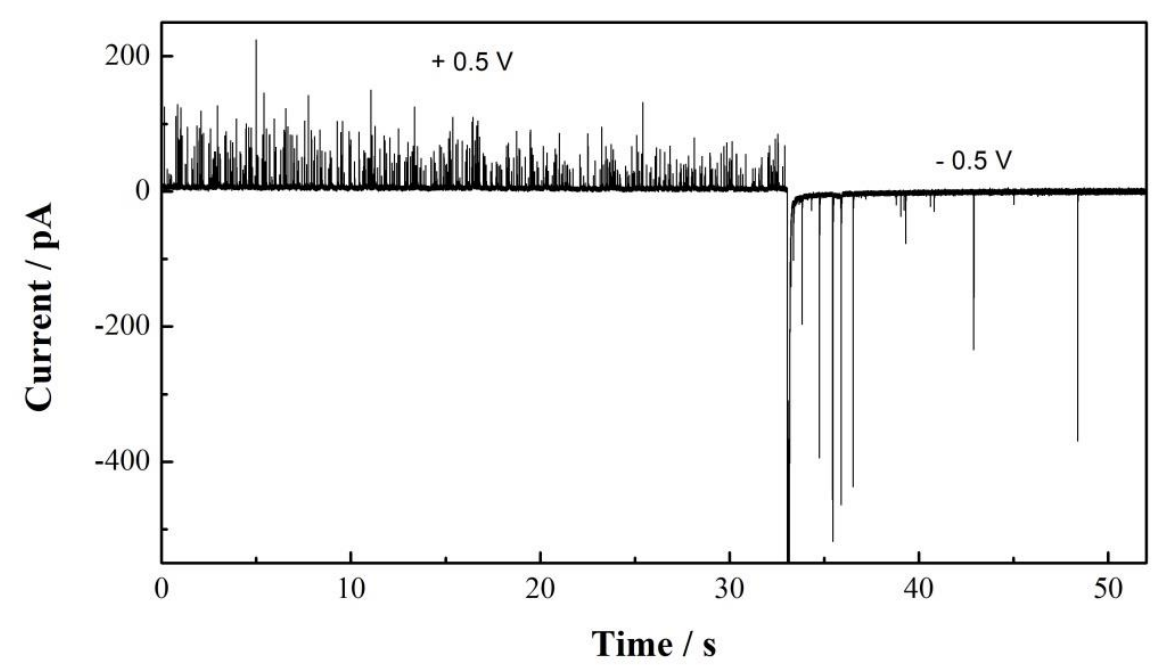

Figure S5. Current-time responses at oxidation and reduction potential of $\pm 0.5 \mathrm{~V}$ without PB buffer. Conditions: $25 \mathrm{pM} \mathrm{Ag} \mathrm{NPs,} 10 \mathrm{mM} \mathrm{NaNO}_{3}$, the sampling rate is $10 \mathrm{kHz}$ and the low-pass filter frequency was $3 \mathrm{kHz}, r_{\mathrm{NP}}=15 \mathrm{~nm}, a=100 \mathrm{~nm}, E=0.5 \mathrm{Vvs} . \mathrm{Ag} / \mathrm{AgCl}$ 


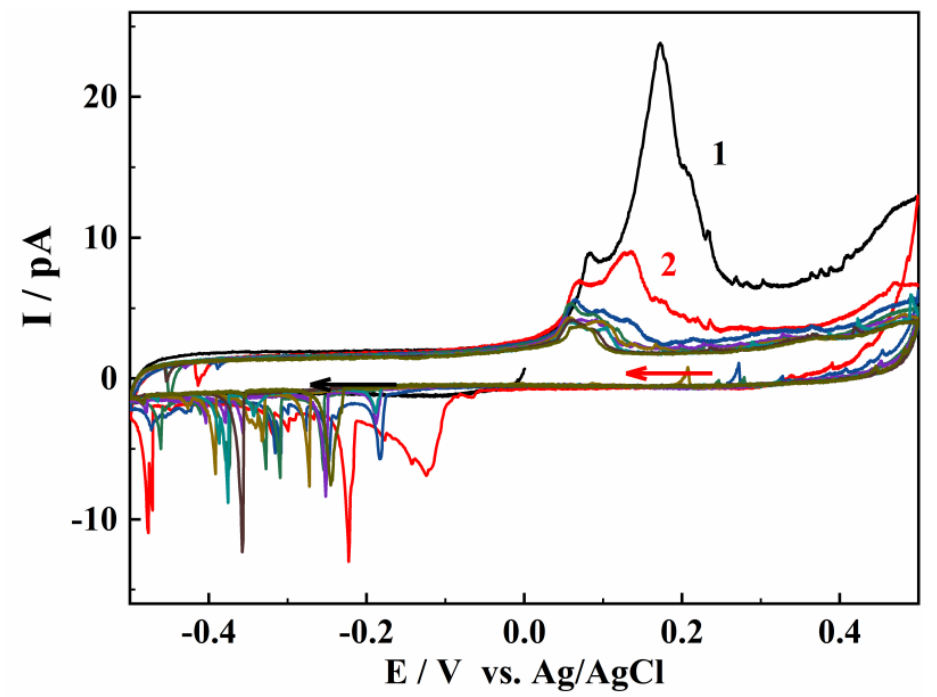

Figure S6. Successive cyclic voltammograms of AgNPs inside carbon nanopipettes at a scan rate of 0.1 V/s. Solution conditions are the same of that in Figure S3. 

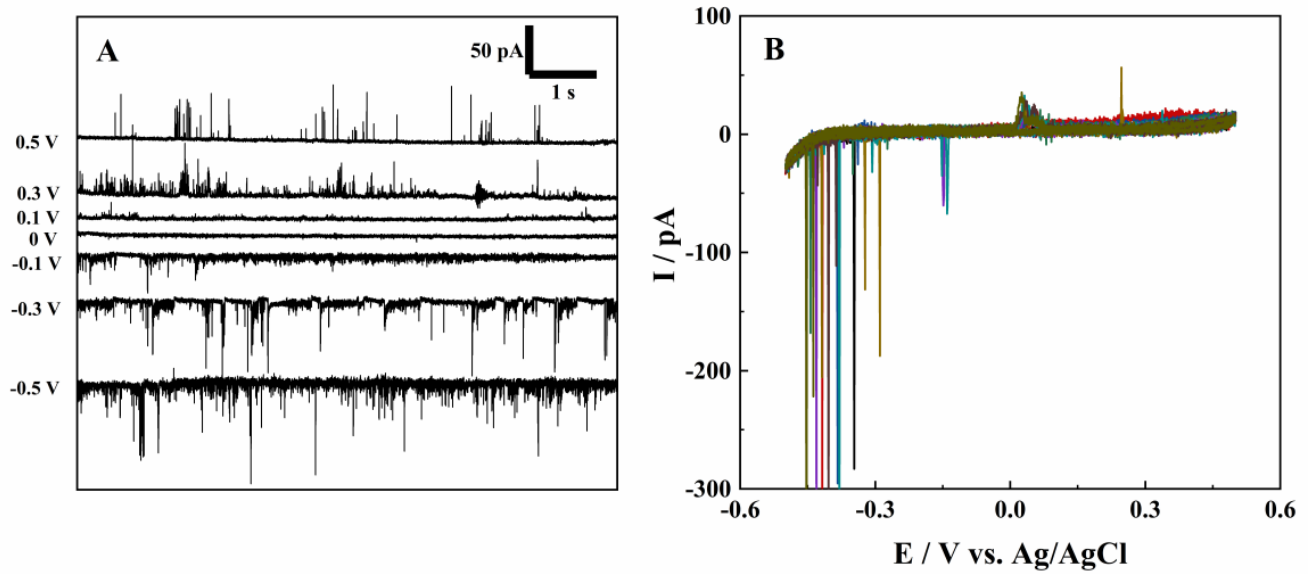

Figure S7. (A) Successive current-time recording of $\mathrm{Ag}^{+}$reduction at different potentials from -0.5 to 0.5 $\mathrm{V}$, the sampling rate was $10 \mathrm{kHz}$ and the low-pass filter frequency was $3 \mathrm{kHz}$. (B) Cyclic voltammograms of $\mathrm{AgNO}_{3}$ in CNP. The solution is $20 \mu \mathrm{M} \mathrm{AgNO}_{3}$ containing $10 \mathrm{mM} \mathrm{NaNO}_{3}$ and $10 \mathrm{mM} \mathrm{PB}, a=100 \mathrm{~nm}$, scanning rate is $0.1 \mathrm{~V} / \mathrm{s}$. 


\section{References}

(1) Liu, J.; Kvetny, M.; Feng, J.; Wang, D.; Wu, B.; Brown, W.; Wang, G., Langmuir 2012, 28, 1588-1595.

(2) Liu, J.; Wang, D.; Kvetny, M.; Brown, W.; Li, Y.; Wang, G., Langmuir 2013, 29, 8743-8752.

(3) Yu, Y.; Noel, J. M.; Mirkin, M. V.; Gao, Y.; Mashtalir, O.; Friedman, G.; Gogotsi, Y., Anal. Chem. 2014, 86, 3365-72.

(4) Wang, Y.; Kececi, K.; Mirkin, M. V.; Mani, V.; Sardesai, N.; Rusling, J. F., Chem. Sci. 2013, 4, 655-663.

(5) Hafez, M. E.; Ma, H.; Peng, Y. Y.; Ma, W.; Long, Y. T., J. Phys. Chem. Lett. 2019, 10, 3276-3281. 